Gut and Liver, Vol. 10, No. 5, September 2016, pp. 657-658

\title{
How Can We Propagate the National Endoscopy Quality Improvement Program and Improve the Quality of Endoscopic Screening?
}

\author{
Dong-Hoon Yang \\ Department of Gastroenterology, Asan Medical Center, University of Ulsan College of Medicine, Seoul, Korea
}

See "National Endoscopy Quality Improvement Program Remains Suboptimal in Korea" by Jae Myung Cha, et al. on page 699, Vol. 10. No. 5, 2016

The National Cancer Screening Program (NCSP) has been provided since 1999 in Korea and the annual surveys for National Cancer Screening showed gradual increase in both the lifetime screening rates and screening rates with recommendation of gastric and colorectal cancers. ${ }^{1}$ In addition to the nationwide propagation of the screening program, the improvement in the endoscopic quality is critical to reduce the mortality related to gastric and colorectal cancers and to minimize the probability of endoscopy-related complications. The National Endoscopy Quality Improvement Program (NEQIP) has been developed to improve the quality of endoscopic screening program of the NCSP. ${ }^{2}$ NEQIP includes various factors related to quality of instruments in the endoscopy units, endoscopic procedures, endoscopic sedation, endoscopic reprocessing, and screening outcomes, as well as qualification of endoscopists. ${ }^{2}$

In the original paper of Gut and Liver, titled as "National endoscopy quality improvement program remains suboptimal in Korea," Cha et al. ${ }^{3}$ reported the results of survey to investigate the nationwide data about the endoscopic capacity, endoscopic sedation, and reprocessing of the endoscopes. A total of 547 physicians, including 342 gastroenterologists, responded in this web-based survey. Although the total response rate was low (18.8\%), this survey provides some important insights related to nationwide screening endoscopy. ${ }^{3}$

First, given that the typical waiting time for screening esophagogastroduodenoscopy and colonoscopy was less than 4 weeks in more than $90 \%,{ }^{3}$ the endoscopic capacity of NCSP can be considered appropriate relative to the current endoscopic screening rates. However, as the screening rate with recommendations has increased gradually since $2004,{ }^{1}$ the current endoscopic capacity may not be sufficient for the increased endoscopic demand in near future. As expected, according to the current survey, the insufficient financial reimbursement is considered as a major obstacle to accommodate the endoscopic demand in their clinical practice. ${ }^{3}$

The survey results also showed that the performance and understanding in endoscopy reprocessing are still suboptimal in spite of the application of quality improvement program since 2009. ${ }^{3}$ As the authors suggested, understanding of NEQIP and knowledge about the reprocessing are necessary to improve the performance of endoscopy reprocessing. However, the results of this survey did not assess the burden and cost of the individual endoscopists or endoscopy units that would require to improve the endoscopy reprocessing. According to another survey about the burden and cost related to the NEQIP, ${ }^{4} 52 \%$ of the responders answered that they were burdened by preparing the audit for NEQIP and needed to pay high costs for additional equipment, devices, and medicines to meet the quality standards that were suggested by NEQIP. The responders also pointed out that the incentives would be important for successful implementation of NEQIP in Korea. ${ }^{4}$

Interestingly, according to the current survey, the responded physicians expected the benefits of NEQIP for their patients, but were concerned about the potentially negative effects of NEQIP in their clinical practice and reimbursement. Moreover, probably because of the gaps between the current NEQIP criteria and the real clinical practice, over $80 \%$ of respondents wanted simplified NEQIP criteria that would be more easily applicable in their practice.

Therefore, to improve the quality of endoscopic screening by

Correspondence to: Dong-Hoon Yang

Department of Gastroenterology, Asan Medical Center, University of Ulsan College of Medicine, 88 Olympic-ro 43-gil, Songpa-gu, Seoul 05505, Korea

Tel: +82-2-3010-5809, Fax: +82-2-3010-6157, E-mail: dhyang@amc.seoul.kr pISSN 1976-2283 eISSN 2005-1212 http://dx.doi.org/10.5009/gnl16349

@ This is an Open Access article distributed under the terms of the Creative Commons Attribution Non-Commercial License (http://creativecommons.org/licenses/by-nc/4.0) which permits unrestricted non-commercial use, distribution, and reproduction in any medium, provided the original work is properly cited. 
propagating the NEQIP, the Korean Society of Gastrointestinal Endoscopy should try to minimize the gap between the NEQIP criteria and the real clinical practice. Considering the importance of the propagation of NEQIP in the clinical practice of each endoscopy unit, stepwise expansion of NEQIP criteria may help to minimize the financial barrier for the screening endoscopy units and psychologic resistance for the endoscopists. In addition, the improvement in the reimbursement system related to the endoscopy is another important step to enhance the quality of endoscopic screening. Considering the unrealistically low cost for endoscopy in Korea, the incentive reimbursement for the qualified screening endoscopy will act as a positive financial feedback for the endoscopy units to uptake the NEQIP in their clinical practice.

\section{CONFLICTS OF INTEREST}

No potential conflict of interest relevant to this article was reported.

\section{REFERENCES}

1. Suh M, Choi KS, Park B, et al. Trends in cancer screening rates among Korean men and women: results of the Korean National Cancer Screening Survey, 2004-2013. Cancer Res Treat 2016;48:110.

2. Cha JM. Quality improvement of gastrointestinal endoscopy in Korea: past, present, and future. Korean J Gastroenterol 2014;64:320-332.

3. Cha JM, Moon JS, Chung IK, et al. National endoscopy quality improvement program remains suboptimal in Korea. Gut Liver 2016;10:699-705.

4. Cho YK, Moon JS, Han DS, et al. Feedback survey of the effect, burden, and cost of the National Endoscopic Quality Assessment Program during the past 5 years in Korea. Clin Endosc. Epub 2016 Mar 2. http://dx.doi.org/10.5946/ce.2015.113. 